\title{
EVALUATING THE TECHNICAL RESOURCE OF MEDIUM AND LARGE CALIBER ARTILLERY WEAPONRY USING THE STANDARD OBJECTIVE FUNCTION
}

\author{
Stelian POPESCU \\ “Nicolae Bălcescu” Land Forces Academy, Sibiu, Romania \\ fam_popes@yahoo.com
}

\begin{abstract}
The technical resource of artillery weapons can be highlighted, in an original way, with the help of a function called by the author standard objective function. In this respect, an original mathematical model thereof was defined and is presented. Consistent with the values achieved in time by the standard objective function qualitative information on the technical resource assessment of artillery mouths is provided. The model developed enables qualitative assessments concerning normal operation or reaching the critical value of the mouth. The defining element of this approach is the diagnosis parameters.
\end{abstract}

Keywords: standard objective function, mathematical model, technical resource, diagnosis parameters

\section{The Technical Resource (Life Span)}

The technical resource of the mouth $\left(\mathrm{R}_{\text {th.g.f }}\right)$ stands for the calendar duration of operation until reaching the critical technical condition or discarding (recast).

The critical technical condition of artillery weaponry corresponds to a situation where it no longer performs the technical parameters designed during operation and training and the safety and security of personnel during fire drills is compromised. Artillery mouths are composed of a plurality of parts, different from a constructive and a functional point of view. The resource is specific to each piece (part) until it reaches the critical condition.

The critical technical condition of the piece is the technical condition where it can no longer perform the functions and having it reconditioned is technically impossible or economically unreasonable.

The technical resource of the part $\left(r_{p}\right)$ is defined as the working time (operating time, number of shots fired) until reaching the critical technical condition.

The technical resources of the parts, subassemblies and mouthsare random sizes whose values will differ according to the laws of variation of objective functions that define them and the evolution of these functions in relation to a standard function declared.

Building a mouth that be made up of parts with identical resources is highly difficult, practically impossible and it cannot be economically justified. In most cases we will have mouths with a $R_{\text {th.g.f }}$ resource, which will embed in its major components $r_{p}$ resources with: $r_{p} \gg R_{\text {th.g.f. }}$

Practically, for a mouth to be able to perform its technical resource it is extremely important to establish (name) the optimum sizes of resources for its subassemblies as well as the relation between these resources and the resource of the mouth. 
In relation to the value of technical resources, the classification of medium and large caliber artillery subassemblies mouths is as presented in Figure 1.

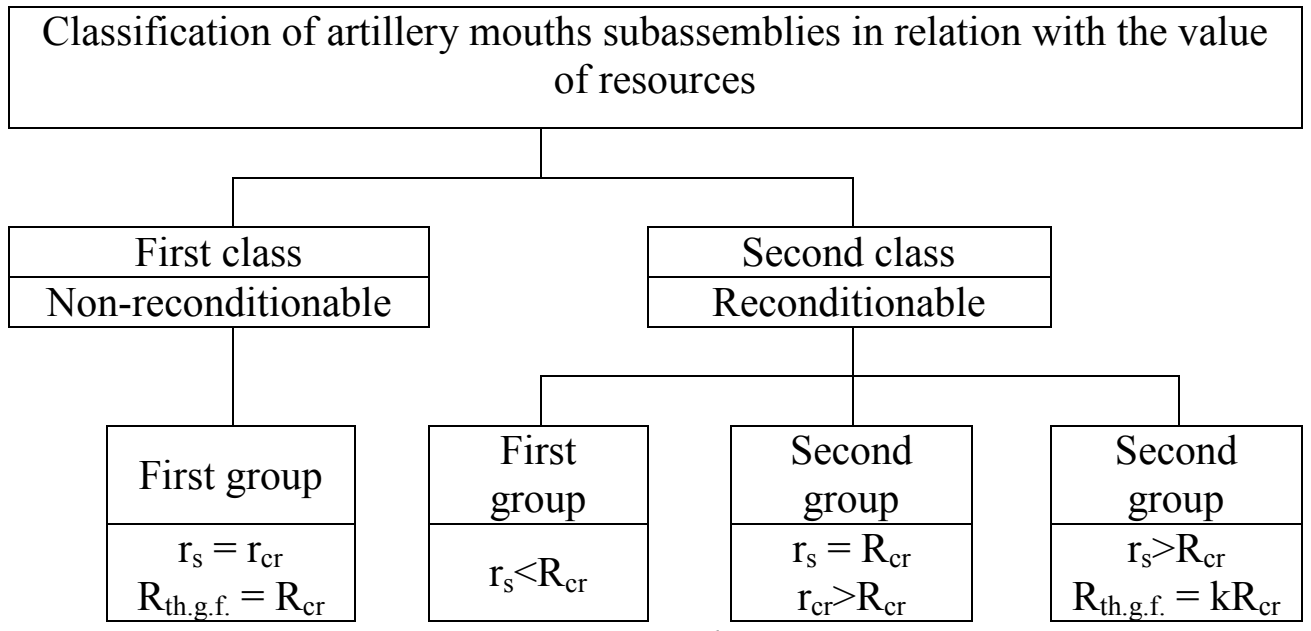

Figure 1

In Figure 1 the following sizes were defined:

$\mathbf{r}_{\mathbf{s}}=$ subassembly resource;

$\mathbf{r}_{\mathbf{c r}}=$ the critical resource of the subassembly;

$\mathbf{R}_{\mathbf{c r}}=$ the critical resource of the mouth;

$\mathbf{k}=$ multiplying ratio.

For all second class subassemblies the following condition must be observed:

$\mathrm{r}_{\mathrm{s}}<\mathrm{r}_{\mathrm{cr}}$

this being in fact the condition that subassemblies can still be reconditioned (the parts can be technically reconditioned in order to fulfill the functions they were designed for and reused for as long as their technical resource has not reached its critical value).

\section{The Evolution in Time of Artillery Weapons' Objective Functions}

Depending on the way objective functions of main and secondary subassemblies of a mouth were defined, it was noticed that the only function that undergoes changes (transformations) in the process of operation of the artillery weaponry is the objective function for level III secondary subassemblies.

The objective function for level III secondary subassemblies, $\mathrm{F}_{\mathrm{obj}}^{\mathrm{III}}$, is calculated using the mathematical relation (3):
$\mathrm{F}_{\text {ob j }}^{\text {III }}=\sum_{\mathrm{i}=1}^{\mathrm{n}} \mathrm{w}_{\mathrm{qlkji}} \cdot \mathrm{p}_{\mathrm{qlkji}}$

where:

$\mathrm{w}_{\mathrm{qlkji}}$ - the weight given to the iparameter within the diagnosis process of the level III secondary subassembly under analysis $(0<$ $\left.\mathrm{w}_{\mathrm{qlkji}} \leq 1\right)$;

$\mathrm{p}_{\mathrm{qlkji}}$ - the score awarded to the iparameter within the diagnosis process according to the diagnosis sheet of the level III secondary subassembly under analysis $\left.\mathrm{p}_{\mathrm{qlkji}}=\{1,2,3\}\right)$.

This function is temporal, as deriving from relation (4).

$\mathrm{F}_{\mathrm{obj}}^{\mathrm{III}}=\mathrm{F}_{\mathrm{obj}}^{\mathrm{III}}(\mathrm{t})$

The evolution in time of this function is determined by the values of the scores awarded to diagnosis parameters, $\mathrm{p}_{\mathrm{qlkji}}(\mathrm{t})$, values that can be established in relation with the way diagnosis parameters vary, $\mathrm{p}_{\text {aram.d }} \mathrm{d}_{\mathrm{qkji}}(\mathrm{t})$.

This way, the relation that defines $\mathrm{F}_{\mathrm{obj}}^{\mathrm{III}}(\mathrm{t})$ will have the form (5):

$\mathrm{F}_{\text {obj }}^{\text {III }}(\mathrm{t})=\sum_{\mathrm{i}=1}^{\mathrm{n}} \mathrm{w}_{\text {qlkji }} \cdot \mathrm{p}_{\text {qlkji }}(\mathrm{t})$ 
The calculation and time variation of the using the expression given by (6) objective function of artillery weaponry,

$$
\mathrm{F}_{\mathrm{ob}}^{\mathrm{qf}}=\sum_{\mathrm{q}=1}^{\mathrm{s}} \mathrm{w}_{\mathrm{q}}^{\mathrm{p}}\left[\sum_{\mathrm{l}=1}^{\mathrm{v}} \mathrm{w}_{\mathrm{ql}}^{\mathrm{I}}\left[\sum_{\mathrm{k}=1}^{\mathrm{t}} \mathrm{w}_{\mathrm{qlk}}^{\mathrm{II}}\left[\sum_{\mathrm{j}=1}^{\mathrm{m}} \mathrm{w}_{\mathrm{qlkj}}^{\mathrm{III}}\left[\sum_{\mathrm{i}=1}^{\mathrm{n}} \mathrm{w}_{\mathrm{qlkji}} \cdot \mathrm{p}_{\mathrm{qlkji}}\right]\right]\right]\right]
$$

can be performed only after having calculated and established the time variations of level III secondary objective functions.

For this it is enough, according to (5), to know the values taken in time by the $\mathrm{p}_{\mathrm{qlkji}}(\mathrm{t})$ functions, values that depend on the evolution in time of diagnosis parameters, $p_{\text {aram. }} \mathrm{d}_{q \mathrm{kji}}(\mathrm{t})$ defined according to the diagnosis sheets of each product.

$$
\mathrm{F}_{\mathrm{ob}}^{\text {g.f. }}(\mathrm{t})=\sum_{\mathrm{q}=1}^{\mathrm{s}} \mathrm{w}_{\mathrm{q}}^{\mathrm{p}}\left[\sum_{\mathrm{l}=1}^{\mathrm{v}} \mathrm{w}_{\mathrm{ql}}^{\mathrm{I}}\left[\sum_{\mathrm{k}=1}^{\mathrm{t}} \mathrm{w}_{\mathrm{qlk}}^{\mathrm{II}}\left[\sum_{\mathrm{j}=1}^{\mathrm{m}} \mathrm{w}_{\mathrm{qlkj}}^{\mathrm{III}}\left[\sum_{\mathrm{i}=1}^{\mathrm{n}} \mathrm{w}_{\mathrm{qlkji}} \cdot \mathrm{p}_{\mathrm{qlkji}}(\mathrm{t})\right]\right]\right]\right]
$$

3. The Standard Objective Function. Evaluating the Technical Resource Using the Standard Objective Function

Under normal circumstances, when the artillery weaponry is operated correctly, variations in parameters contained in the diagnosis sheets evolve in accordance with the laws determined experimentally or statistically.

For the casewherevariations in diagnosis parameters are the ones considered we can define an objective function of the mouth called the standard objective function, $\mathrm{F}_{\mathrm{ob}}^{\mathrm{g} . \mathrm{f}}$.

For any mouth the evolution in time of the objective function will have a random variation, situated around the standard objective function. When representing graphically this function, we can notice that, in relation with the operating mode of the mouth, the function graph can be

$$
\mathrm{F}_{\mathrm{ob}}{ }^{\mathrm{gf}}(\mathrm{t})
$$

The mathematical expressions for the laws of variation of diagnosis parameters, $\mathrm{p}_{\text {aram. }} \mathrm{d}_{\mathrm{qlkji}}(\mathrm{t})$, can be determined by means of experimental methods or can be statistically assessed based on the data obtained in the operation and maintenance processes of artillery weaponry.

Using (5) and (6) the objective function of a mouth will be determined using the relation (7): 


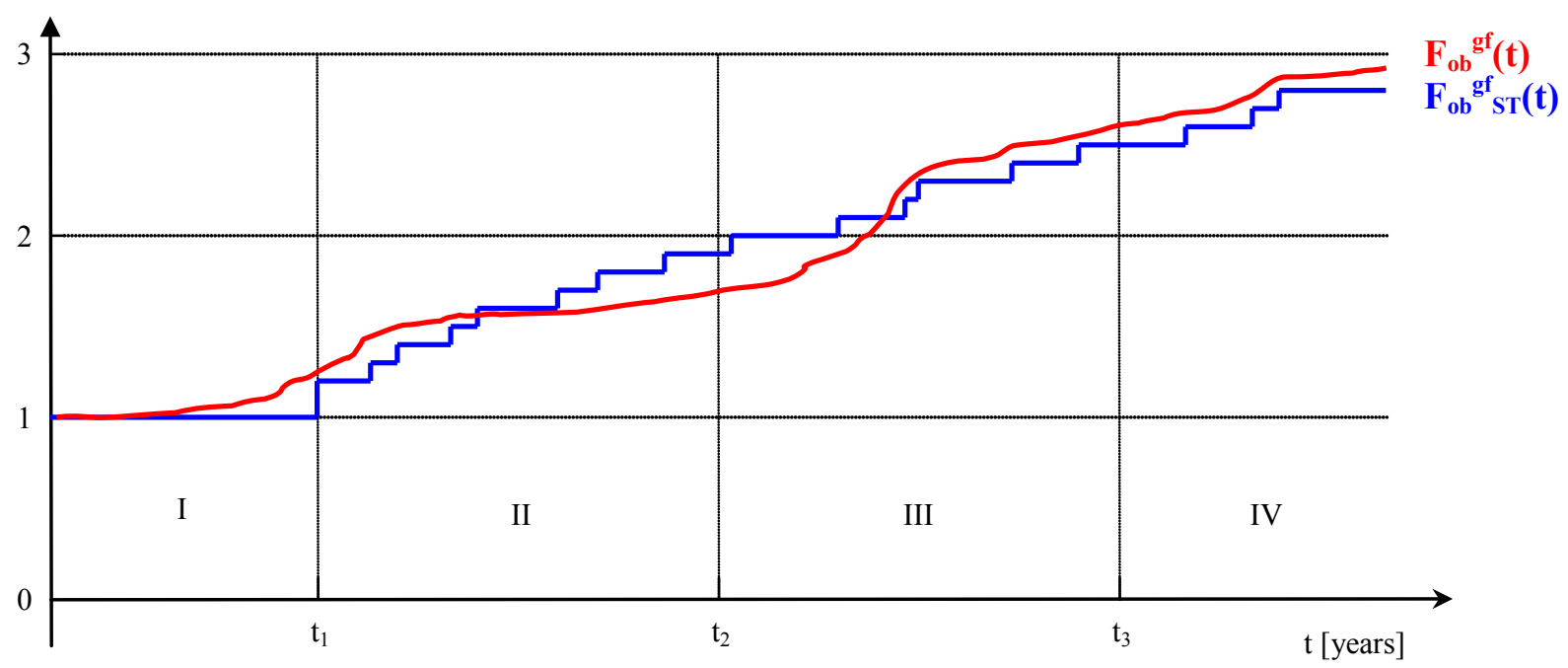

Figure 2 The evolution of the objective function in time of a mouth in relation with the standard function

Consistent with the values achieved overtime by the standard objective function, according to Figure2, four distinct situations may arise, materialized in the four graphical areas I ... IV, which provide qualitative information on the assessment of the technical resource of an artillery mouth:

- $\quad$ area $\mathrm{I}, \mathrm{t} \in\left[0, \mathrm{t}_{1}\right]$, where $\mathrm{F}_{\mathrm{ob}}^{\mathrm{g} \mathrm{b}_{\mathrm{ST}}}(t)=1$ and allp $\mathrm{qlkji}=1$.

The mouth has a very good technical resource and may be further operated safely;

- $\quad$ area II, $\mathrm{t} \in\left(\mathrm{t}_{1}, \mathrm{t}_{2}\right]$, where $1<\mathrm{F}_{\mathrm{ob}}^{\mathrm{g} . \mathrm{f}}(\mathrm{t})<2$ and all $\mathrm{p}_{\mathrm{qlkji}}=\{1,2\}$.

The mouth has a good technical resource and may be further operated without restrictions;

- $\quad$ area III, $\mathrm{t} \in\left(\mathrm{t}_{2}, \mathrm{t}_{3}\right]$, where $1<\mathrm{F}_{\mathrm{ob}}^{\mathrm{g} . \mathrm{f}}(\mathrm{t})<3$ and some $\mathrm{p}_{\mathrm{qlkji}}=3$.

The mouth can be maintained in service with some restrictions or be removed from service and sent to a specialized structure to be repaired. The decision to keep in operation or to have repaired is made after an analysis of risk factors in relation to critical values achieved by certain parameters.

The parameters that reached critical values will be referred to as critical parameters. Critical parameters that influence the decision on maintaining or taking out of service a mouth can be divided into two groups:

- the critical parameters that enable the maintenance into operation of the mouth, called nonhazardous critical parameters (e.g.the state of the protective coatings of the breech, the technical condition of the sponge tire, shield surface integrity etc.) and which may be removed during weaponry operation with specialized forces and means in the unit;

- the critical parameters that demand the decommissioning of the mouth and performing an analysis concerning the possibility of regaining technical status through maintenance interventions or the decommissioning thereof in view of cassation;

- these parameters will be referred to as hazardous critical parameters (e.g. leakage of fluid from the brake cylinder in quantities greater than those allowed, fast speed of returning to the battery with shocks or vibrations, the switch does not ensure complete closure of the barrel, selfdrilling etc.).

The weaponry that needs repairs will be sorted and sent to different maintenance structures, in relation to the complexity of the work that must be performed. Low 
technical level maintenance interventions will be carried out by specialized forces and means in the unit that owns the equipment, medium level maintenance interventions will be performed by specialized forces and means in the next level tactical unit, while high level maintenance operations will be performed with the specialized forces and means available in operational units or forces component headquarters (Special Technical Maintenance Centers). Prior to executing high-level maintenance interventions an economic calculation and a technical analysis are performed in order to prove the efficiency and effectiveness in the execution of these types of interventions.

This area can be defined as the optimum maintenance interventions area for artillery weaponry.

- $\quad$ area $I V, t>t_{3}$, where $F_{o b}^{g . f}(t)=3$

The mouth can no longer be operated. Its use in these conditions endangers those who operate it.

In this case an economic calculation is performed regarding the efficiency of the mouth repair or having it removed from service.
The calculation is performed in a specialized maintenance structure of the Ministry of Defense.

If it is found that the value of the repair exceeds $60 \%$ of the replacement value of the product then it is no longer repaired and is proposed for removal from operation, decommissioning and disposal.

\section{Conclusions}

The evolution intimae of objective functions of artillery weaponry is dependent on the evolution in time of diagnosis parameters, translated into scores awarded to them.

The technical resource of large and medium caliber mouths was highlighted in an original way by means of the standard objective function calculated for each type of mouth.

The value of the objective function of the mouth, at a given moment, offers information on its technical resource, corresponding to the function's value level. Consequently, we can assess the moment of reaching the critical value of the mouth, which makes its operation unsafe or hazardous from the point of view of the security of the personnel operating it.

\section{References}

[1] Bezovsky, I., Fiabilite - theorieetpratique de la surete de fonctionnement, Dunod Publishing House, Paris, 1966.

[2] Cardasevschi, C.,TheFiabilist Diagnosis of Industrial Products, Technical Publishing House, Bucharest, 1990.

[3] Constantin, Gh.;Nuţu, V, Basics of Weapons Systems Reliability, A.T.M. Publishing House, Bucharest 1996.

[4] Lloyd, K.D.; Lipow, M., Reliability: Management, Methods and Mathematics, Hall Inc. Englewood Cliffs, New Jersey.

[5] Manea, C.; Stratulat, M., Reliability and Diagnosis of Vehicles, The Military Publishing House, Bucharest, 1982.

[6] Popescu, St.; Dascălu, V., Calculus of System Reliability, Scientific Conference, "Nicolae Bălcescu" Land Forces Academy, Sibiu, 2002.

[7] Popescu, St.;Dascălu, V., Risk Management in Maintaining Artillery Weaponry, Scientific Conference, "Nicolae Bălcescu” Land Forces Academy, Sibiu, 2005. 